\title{
Suppression of Fluctuating Lift on a Circular Cylinder by Attaching Cylindrical Rings*
}

\author{
Hajime NAKAMURA** \\ ** National Defense Academy \\ 1-10-20 Hashirimizu, Yokosuka-shi, Kanagawa, Japan \\ E-mail: nhajime@nda.ac.jp
}

\begin{abstract}
Omnidirectional reductions in drag and fluctuating forces can be achieved for a circular cylinder subjected to cross-flow by attaching cylindrical rings along its span at an interval of several diameters. In this work, the effects of ring diameter $(D)$, spanwise width $(W)$, and spanwise pitch $(P)$ on vortex shedding suppression were investigated. It was found that the periodicity of the pressure fluctuation on the sides of the cylinder disappeared at higher Reynolds numbers $\left(R e_{d} \geq 20,000\right)$ for the ring configuration with $D / d=1.3, W / d=1$, and $P / d \approx 3$, where $d$ is the base cylinder diameter. In this configuration, the fluctuating lift force was about $1 / 30$ that of a $2 \mathrm{D}$ cylinder due to the suppression of periodic shedding and the reduction of spanwise correlation. The mechanism behind this was explored through flow visualization and particle image velocimetry and is considered to be as follows: A spanwise pressure gradient originating from a stepwise change in diameter induces spanwise flow, which brings the corner vortex to the side of the ring. This promotes laminar-turbulent transition in the shear layer separated from the ring at $R e_{d} \geq$ 20,000 . As a result, the wake behind the ring shrinks significantly, which induces a pair of large transverse vortices just behind the ring edges. Consequently, the formation of two-dimensional spanwise vortices is obstructed, and the periodicity of the vortex shedding is suppressed.
\end{abstract}

Key words: Passive Flow Control, Drag Force, Fluctuating Force, Circular Cylinder, Vortex Shedding, Three-Dimensional Flow

\section{Introduction}

It is well known that a two-dimensional bluff body subjected to cross-flow generates periodic vortex shedding, which sometimes causes unfavorable effects, such as vortex-induced vibration and aeroacoustic noise. Therefore, the use of passive flow control to suppress these effects has been widely investigated as reviewed by Zdravkovich ${ }^{(1)}$. An example applicable to a circular cylinder is the attachment of thin protuberances called "tripping wires" in front of the separation lines, which promote laminar-turbulent transition in the subcritical flow regime $\left(2 \times 10^{4}<R e_{d}<2 \times 10^{5}\right)^{(2)(3)}$.

Vortex shedding can also be suppressed through three-dimensional surface modifications. Bearman and Owen ${ }^{(4)}$ and Owen et al. ${ }^{(5)}$ investigated drag reduction and vortex shedding suppression on a rectangular cylinder and a circular cylinder by introducing wavy separation lines. That is, a sinusoidal wave-shaped front face was added to the cylinder with a rectangular cross-section, and a sinusoidal wave-shaped axis was added to the cylinder with a circular cross-section. As a result, the drag coefficients were reduced by $30-47 \%$ compared to the unmodified cylinders, even though the amplitude of the waviness was small: The ratio of the peak-to-peak wave height to the wavelength was approximately 
0.1 .

Although these modifications dramatically reduce drag and suppress vortex shedding, they are only effective for a given flow direction relative to the cylinder. A method that is effective for all flow directions was proposed by Owen et al. ${ }^{(5)}$ in which hemispherical bumps were attached in a spiral pattern to the surface of a circular cylinder. This geometry resembles "helical strikes"(6) and "helical wires"(7), which have often been applied to circular cylinders to suppress flow-induced vibration. However, the addition of helical strikes and helical wires resulted in an increase in the drag force ${ }^{(1)}$. In contrast, the geometry proposed by Owen et al., together with vortex shedding suppression, reduced the drag coefficient by $25 \%$.

Ahmed et al. ${ }^{(8)(9)}$ studied the flow around a wavy circular cylinder, the diameter of which changed sinusoidally along its span. In this configuration, streamwise vortices were observed near the geometric nodes of the cylinder; however, the drag reduction was small (9\% maximum) for an aspect ratio (axial length divided by mean diameter) of about 10 . Lam et al. ${ }^{(10)}$ investigated a similar wavy circular cylinder with a larger aspect ratio (about $27)$; in this experiment, the drag coefficient was reduced by up to $20 \%$. Zhang and Lee ${ }^{(11)}$ investigated the flow field behind a wavy circular cylinder using two-dimensional particle image velocimetry (PIV) and observed well-organized streamwise vortices with alternating positive and negative vortices.

The present author ${ }^{(12)(13)}$ proposed adding cylindrical rings to the circular cylinder (step changes in the diameter of the cylinder), as shown in Fig. 1, as a means of three-dimensional surface modification. The advantages of this geometry are axial symmetry (omnidirectionality) and manufacturing simplicity. The drag force $F_{D}$ was lowest (reduced by $15 \%$ ) when $D / d \approx 1.3, W / d \approx 1.0$, and $P / d \approx 6$ (at $R e_{d} \approx 30,000$ ), although the projected area increased when more rings were attached. Figure 2 shows the drag reduction caused by the addition of the rings. As shown in this figure, the drag reduction was significant above a Reynolds number of about 10,000; this is attributed to the transition in the shear layer separated from the ring, as described later. Surface pressure fluctuation was also significantly reduced by adding the rings, which is likely to suppress vortex induced vibration and/or aeroacoustic noise. Lim and Lee ${ }^{(14)}$ controlled the flow behind a circular cylinder by attaching thin O-rings arranged at $0.0167 \leq P / d \leq 2$, the configuration of which has some similarity to the ringed cylinder proposed here. In this case, the drag coefficient $C_{D}$ was reduced by $9 \%$ maximum at $R e_{d}=120,000$.

This paper focuses on the suppression of vortex shedding by the addition of cylindrical rings. First, the optimum ring configuration for vortex shedding suppression was

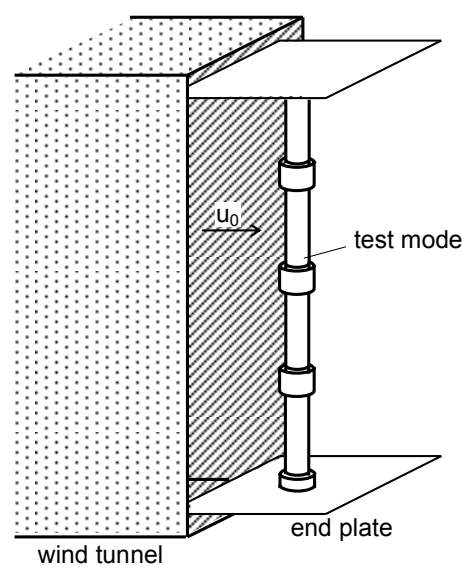

(a) schematic diagram

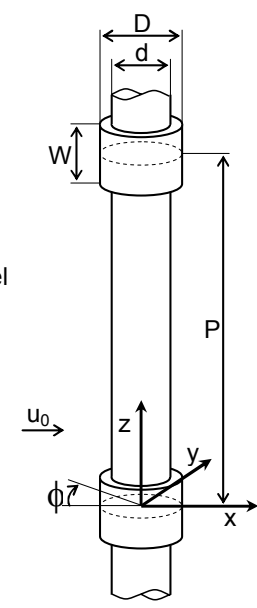

(b) symbols

Fig. 1 Ringed cylinder test setup 


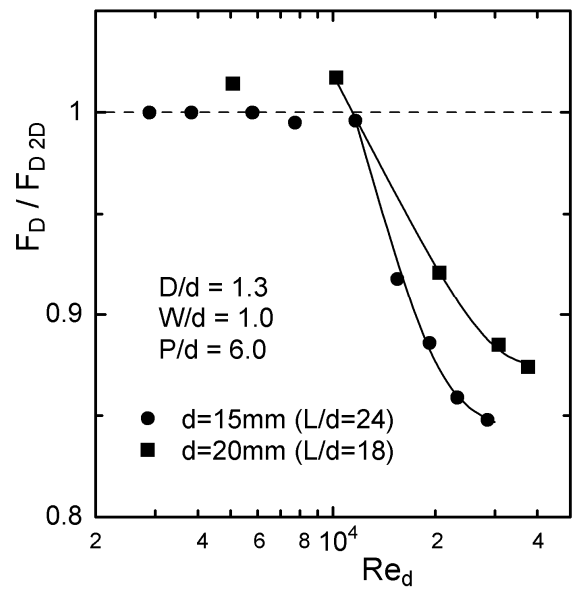

(a) drag force, $F_{D} / F_{D 2 D}$

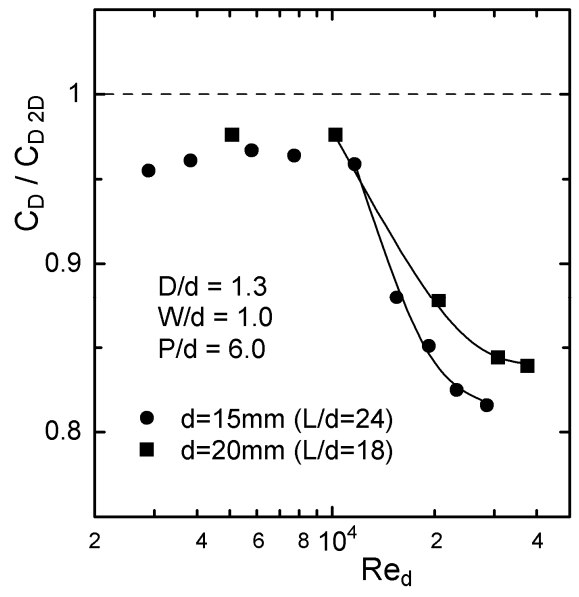

(b) drag coefficient, $C_{D} / C_{D 2 D}$

Fig. 2 Effect of Reynolds number on drag for the optimum ring configuration

determined by measuring the surface pressure fluctuation for various ring configurations. Also, PIV and flow visualization were used to explore the mechanism behind the vortex shedding suppression.

\section{Nomenclature}

$A$ : projected area to the flow $=d L+n(D-d) W \mathrm{~m}^{2}$

$C_{D}:$ drag coefficient $=F_{D} /\left(0.5 \rho u_{0}^{2} A\right)$

$C_{L}:$ lift coefficient $=F_{L} /\left(0.5 \rho u_{0}^{2} A\right)$

$C_{p}$ : pressure coefficient $=\left(p-p_{0}\right) /\left(0.5 \rho u_{0}^{2}\right)$

$D$ : diameter of ring $\mathrm{m}$ or $\mathrm{mm}$

$d$ : diameter of circular cylinder $\mathrm{m}$ or $\mathrm{mm}$

$F_{D}, F_{L}$ : drag and lift forces $\mathrm{N}$

$f, f_{s}$ : frequency, vortex shedding frequency $\mathrm{Hz}$

$L:$ axial length of cylinder $\mathrm{m}$ or $\mathrm{mm}$

$n:$ number of rings

$P$ : spanwise pitch of rings $\mathrm{m}$ or $\mathrm{mm}$

$p:$ static pressure on a wall $\mathrm{Pa}$

$p_{0}:$ static pressure at freestream $\mathrm{Pa}$

$R:$ cross-correlation coefficient

$R e_{d}:$ Reynolds number $=u_{0} d / v$

$S t_{d}:$ Strouhal number $=f_{s} d / u_{0}$

$t:$ time $\mathrm{sec}$

$u, u_{0}:$ velocity, freestream velocity $\mathrm{m} / \mathrm{s}$

$W:$ spanwise width of ring $\mathrm{m}$ or $\mathrm{mm}$

$x, y, z:$ streamwise, cross-stream, and spanwise coordinates (see Fig. 1)

$\phi:$ angle from the forward stagnation point ${ }^{\circ}$ subscripts

$v, \rho$ : kinematic viscosity $\left(\mathrm{m}^{2} / \mathrm{s}\right)$ and density of fluid $\left(\mathrm{kg} / \mathrm{m}^{3}\right)$

$2 D:$ two-dimensional cylinder

( ) : time-averaged value

( )' : rms value 


\section{Experimental Apparatus}

Figure 1 shows a schematic of the test setup. The test cylinder was fabricated from acrylic resin pipe and had a diameter $d$ of 20 $\mathrm{mm}$ and a length $L$ of $360 \mathrm{~mm}$. Acrylic resin cylindrical rings were attached to the cylinder. The ring diameter $(D)$, spanwise width $(W)$, and spanwise pitch $(P)$ were varied in the ranges of $1.2 \leq D / d \leq 1.5,0.5 \leq W / d \leq 2.0$, and $2 \leq P / d \leq 9$. The test cylinder was placed at the exit of the wind tunnel, which has a $400 \mathrm{~mm}$ high and $150 \mathrm{~mm}$ wide working section. The freestream velocity $u_{0}$ ranged from 8 to 30 $\mathrm{m} / \mathrm{s}$, resulting in a Reynolds number (based on $d$ ) between 10,000 and 40,000. The turbulence intensity of the freestream was approximately $0.5 \%$.

The surface pressure fluctuation was measured using two pressure transducers (PD104K-10K, Toyoda), which were mounted within the test cylinder. Two $1.5 \mathrm{~mm}$ diameter pressure holes, each connected to a transducer, were placed directly across from each other at the mid-span of the cylinder, as shown in Fig. 3. These holes were used to simultaneously measure the fluctuating pressure on both sides of the cylinder $\left(\phi= \pm 90^{\circ}\right)$. The resonance frequency of the pressure holes was approximately $2 \mathrm{kHz}$, which means that frequencies below $400 \mathrm{~Hz}$ could be measured without resonance effects. The pressure distribution around the cylinder could also be measured by rotating the cylinder around its axis, thereby adjusting the circumferential position of the pressure holes.

The flow field behind the cylinder was measured using two-dimensional PIV. Oil mist with an average diameter of about $1 \mu \mathrm{m}$ was seeded into the air flow. The mist was illuminated at $15 \mathrm{~Hz}$ by a double-pulse laser sheet (Solo PIV II-15, New Wave Research), which was oriented in the $x-y$ or $x-z$ plane. A camera (XS-3, IDT) with a resolution of $1280 \times 1028$ pixels and a spatial resolution of $0.07 \mathrm{~mm} /$ pixel was used to record the flow; the interrogation window was $32 \times 32$ pixels. The mean velocity field was calculated by averaging 500 instantaneous flow fields.

The flow around the cylinder was visualized using the smoke-wire method and the surface oil-film technique. For the smoke-wire method, paraffin oil was applied to a tightened $0.1 \mathrm{~mm}$ Nichrome wire; when the wire was heated, smoke (paraffin mist) was generated and entrained in the air-flow. The smoke flow, which was illuminated by metal halide lamps (HVC-SL, Photron), was recorded using a high-speed video camera (XS-3, IDT) at $600 \mathrm{frames} / \mathrm{s}$.

\section{Suppression of Fluctuating Lift}

\subsection{Surface pressure fluctuation}

The fluctuating force acting on the cylinder was investigated by measuring the surface pressure fluctuations at both sides of the cylinder $\left(\phi= \pm 90^{\circ}\right)$. Figure 4 displays time traces of the pressure coefficient $C_{p}$ at $R e_{d}=31,000$, and Figure 5 shows the corresponding power spectrums. For the 2D cylinder in Fig. 4(a), the pressures fluctuated vigorously in an alternating sinusoidal pattern corresponding to periodic shedding, which caused a large fluctuating lift to act on the cylinder. The power spectrum (the dotted line in Fig. 5) had a dominant peak at the shedding frequency of $S t_{d}=f_{s} d / u_{0}=0.20$.

The addition of the rings weakened the fluctuations, as shown in Fig. 4(b-h), which largely depended on the ring configuration. For the most effective ring configuration of $D / d$ $=1.3, W / d=1.0$, and $P / d=6$ (Fig. $4[\mathrm{~b}])$, the values of $C_{p}{ }^{\prime}\left(\mathrm{RMS}\right.$ of $\left.C_{p}\right)$ were 0.121 and 
(a) 2D cylinder
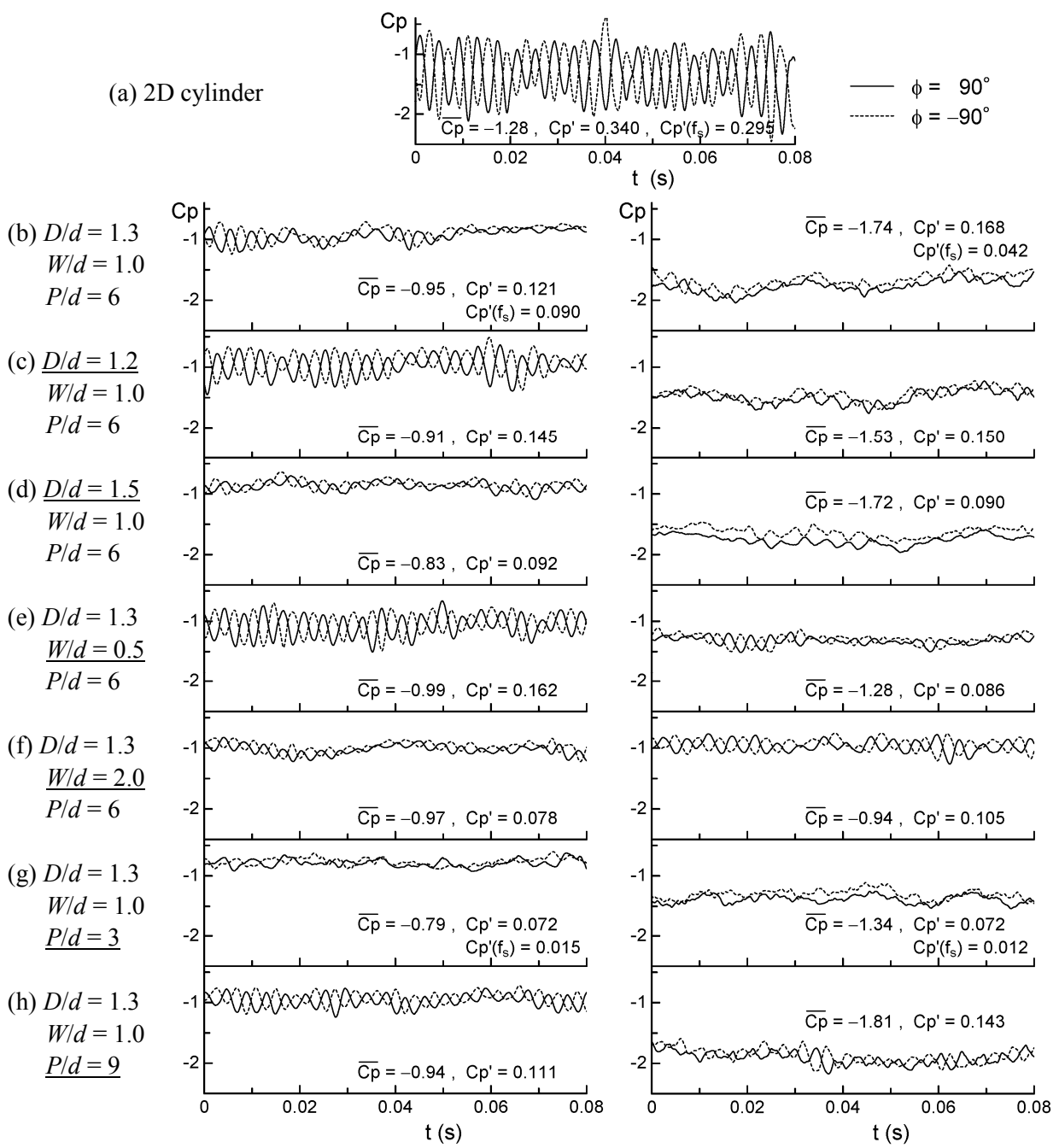

Fig. 4 Surface pressure fluctuation on the sides of the cylinder at $\phi= \pm 90^{\circ}\left(d=20 \mathrm{~mm}, u_{0}=24 \mathrm{~m} / \mathrm{s}\right.$, $\left.R e_{d}=31,000\right) ;$ left - on the base cylinder $(z=P / 2)$, right - on the ring $(z=0)$

0.168 for the base cylinder and ring, respectively, which are about $1 / 3$ and $1 / 2$ the values for the $2 \mathrm{D}$ cylinder. The value of $C_{p}{ }^{\prime}\left(f_{s}\right)$, which is defined within a frequency band of $f_{s} \pm 10 \%$, was much smaller for the ring (0.042) since the signal was mainly comprised of a low-frequency fluctuation unrelated to periodic shedding. Despite a significant reduction in fluctuation, a dominant peak still existed in the power spectrum at the shedding frequency (Fig. 5[c], $P / d=6$ ).

For the smaller ring diameter (Fig. $4[\mathrm{c}]$ ) and width (Fig. 4[e]), the ring's effect was less significant. In contrast, for the smaller ring pitch (Fig. $4[\mathrm{~g}], P / d$ $=3$ ), the periodic fluctuation was effectively suppressed on both the base cylinder and the ring. In this case, the (a)

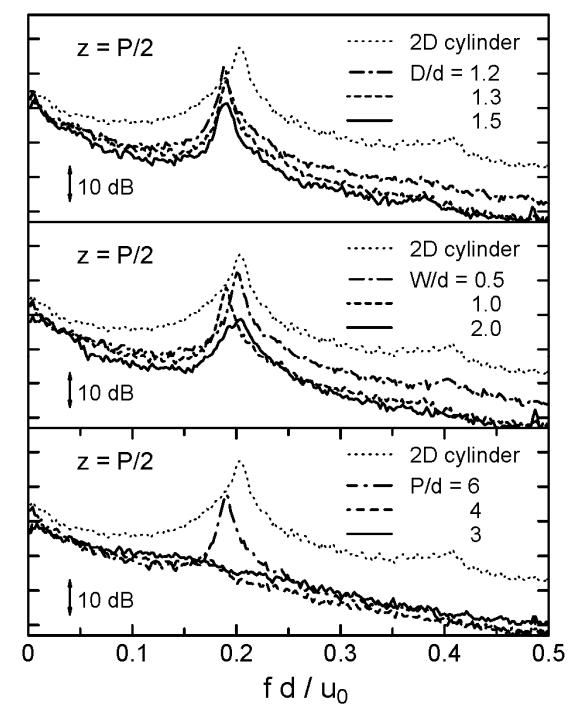

Fig. 5 Power spectrum of the fluctuating pressure coefficient at $\phi=90^{\circ}$ on the base cylinder $(z=P / 2)$, corresponding to Fig. 4 ;

(a) effect of $D / d$, (b) effect of $W / d$, (c) effect of $P / d$ 


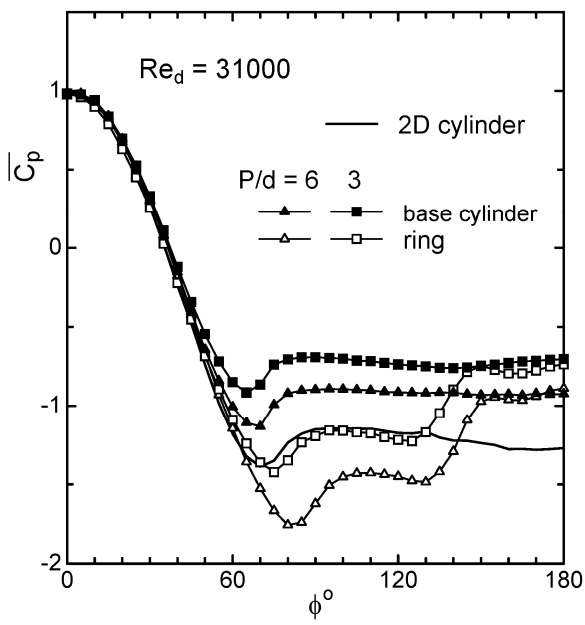

(a) time-averaged distribution

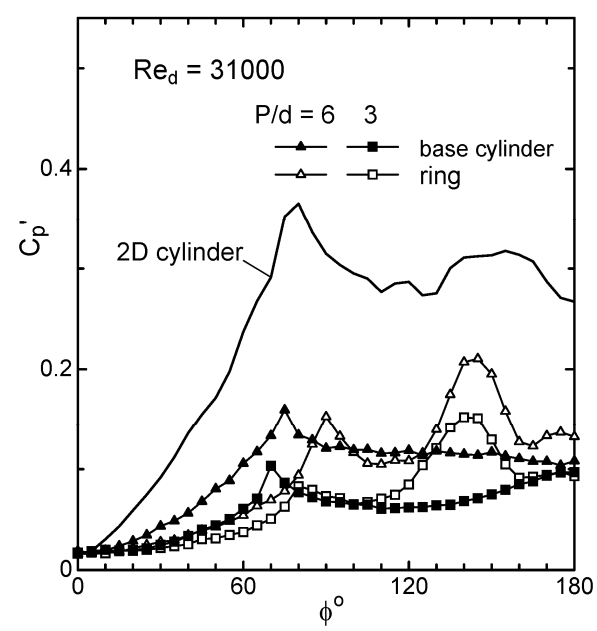

(b) fluctuating pressure coefficient

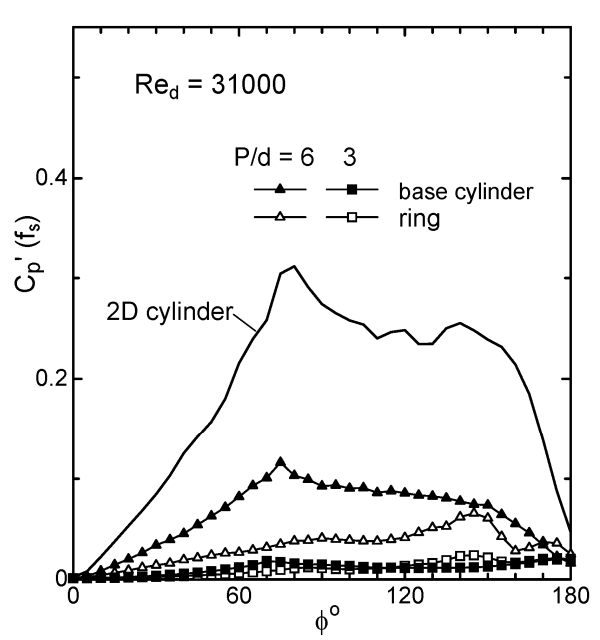

(c) fluctuating pressure coefficient within a frequency band of $f_{s} \pm 10 \%$

Fig. 6 Pressure distribution around the ringed cylinder at $\operatorname{Re}_{d}=31,000(D / d=1.3, W / d=1)$

power spectrum peak at the shedding frequency disappeared, as shown in Fig. 5(c); this indicates full suppression of periodic shedding for this ring configuration.

\subsection{Pressure distribution}

Figure 6 depicts the pressure distributions around the cylinder at $R e_{d}=31,000$. Figure 6(a) shows the time-averaged pressure coefficient $C_{p}$, and Fig. 6(b) shows the RMS value of the fluctuating pressure coefficient $C_{p}$ '. For the ringed cylinder, the flow separated from the side of the ring (at $\phi=80^{\circ}$ for $P / d=3$; at $\phi=90^{\circ}$ for $P / d=6$ ) and reattached to the rear of the ring (at $\phi=140^{\circ}$ for $P / d=3$; at $\phi=145^{\circ}$ for $P / d=6$ ), forming a separation bubble between the separation and reattachment points (shown later in Figs. 11 and 12). At these points, the values of $C_{p}$ ' were maximums, as shown in Fig. 6(b). Since the formation of separation bubbles on both sides of the ring led to the narrowing of the wake behind the ring, the base suction recovered, unlike the 2D cylinder; this decreased the drag of the ringed cylinder. Although the pressure recovery was more prominent for $P / d=3$ than for $P / d=6$, as shown in Fig. 6(a), the drag reduction was more effective for $P / d=6$ (Ref. [12] and [13]); this was due to the increase in projected area and the forward movement of the separation point for $P / d=3$ (Fig. 6[a]).

Figure $6(\mathrm{c})$ shows the values of $C_{p}{ }^{\prime}\left(f_{s}\right)$, which is the RMS of the fluctuating pressure 
coefficient within a frequency band of $f_{s} \pm 10 \%$. The shedding frequency for $P / d=3$, which has no dominant peak in the power spectrum, was estimated by $f_{s}=S_{t 2 \mathrm{D}} u_{0} / d_{m}$, where $S_{t 2 \mathrm{D}}$ is the Strouhal number for the 2D cylinder and $d_{m}$ is the mean diameter of the ringed cylinder. The values of $C_{p}{ }^{\prime}\left(f_{s}\right)$ are similar to that of $C_{p}$ ' for both the $2 \mathrm{D}$ cylinder and the base cylinder with $P / d=6$, where fluctuations are dominated by periodic shedding. In contrast, for $P / d=$ 3 , the value of $C_{p}{ }^{\prime}\left(f_{s}\right)$ is markedly lower than that of $C_{p}$ ' for both the base cylinder and the ring, where periodic shedding does not occur.

\subsection{Estimation of fluctuating lift coefficient}

The fluctuating lift coefficient acting on a section of the cylinder (the local fluctuating lift coefficient) can be estimated assuming that the pressure fluctuation on both sides of the cylinder $(\phi>0$ and $\phi<0)$ are completely out of phase ${ }^{(15)}$.

$$
C_{L}{ }^{\prime}\left(f_{s}\right) \approx \int_{0}^{\pi} C_{p}^{\prime}\left(\phi ; f_{s}\right) \sin \phi d \phi
$$

Here, $C_{L}$ ' is the RMS fluctuating lift coefficient, and $C_{p}{ }^{\prime}\left(\phi, f_{s}\right)$ is the RMS pressure coefficient at $\phi$ within a band of $f_{s}$. Also, the fluctuating lift can be roughly estimated as follows $^{(16)}$ :

$$
C_{L}{ }^{\prime}\left(f_{s}\right) \approx \sqrt{2\left\{1-R_{p p}\left(\phi= \pm 90^{\circ}\right)\right\}} C_{p}{ }^{\prime}\left(\phi=90^{\circ} ; f_{s}\right),
$$

where $R_{p p}\left(\phi= \pm 90^{\circ}\right)$ is the cross-correlation coefficient of the pressure fluctuation between $\phi=90^{\circ}$ and $-90^{\circ}$ and is calculated as follows:

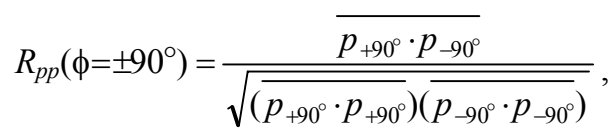

The estimated values of $C_{L}$ ' within a frequency band of $f_{s} \pm 10 \%$ were plotted in Fig. 7 against the Reynolds number. For the ringed cylinder, $C_{L}$ ' was weight-averaged across the base cylinder $(z=P / 2)$ and the ring $(z=0)$. The solid curve in Fig. 7 is an empirical function evaluated by Norberg ${ }^{(15)}$ for a long $2 \mathrm{D}$ cylinder. This curve markedly increases with the Reynolds number in the range of $R e_{d}=4,000-8,000$, at which the vortex formation region approaches the rear of the cylinder. Namely, the effects of vortex-induced vibration and/or aeroacoustic noise become significant at $R e_{d} \geq 10^{4}$.

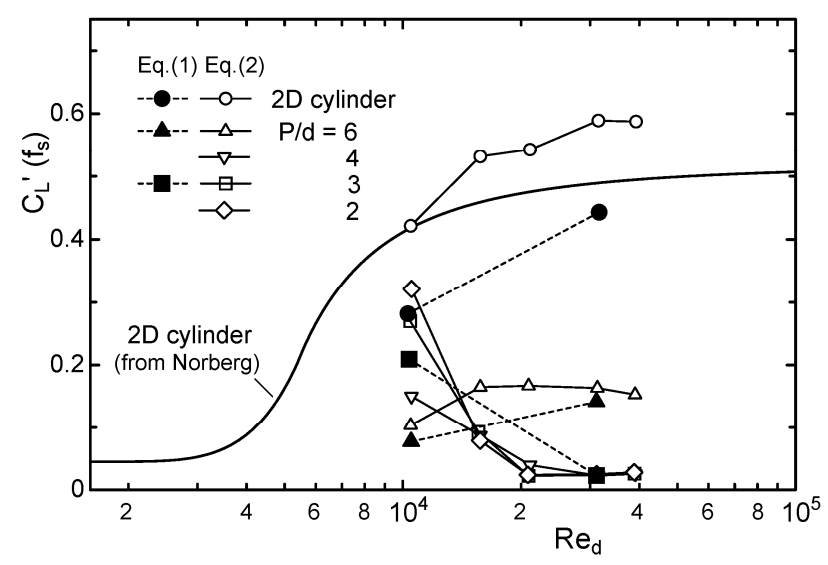

Fig. 7 Fluctuating lift coefficient estimated from the surface pressure fluctuation 
The fluctuating lift coefficient estimated from Eqs. (1) and (2) for the 2D cylinder (solid and open circles in Fig. 7) agrees fairly with the empirical curve, although there are some differences in the experimental conditions, such as aspect ratio and blockage ratio. Incidentally, the value from Eq.(1) maybe an underestimate since the frequency components except for a band of $f_{s}$ are neglected, and the value from Eq.(2) is an overestimate according to Norberg ${ }^{(15)}$.

In contrast, the addition of the ring reduces the fluctuating lift, particularly at $R e_{d} \geq$ 20,000 ; this is due to the transition of the shear layer at $R e_{d} \geq 20,000$, which causes a narrowing of the wake behind the ring. As expected, the fluctuating lift for the smaller ring pitch $(P / d \approx 3)$ almost disappears; its $C_{L}$ ' value is about $1 / 20$ that of the $2 \mathrm{D}$ cylinder.

\subsection{Spanwise correlation}

Assuming that the fluctuation has no phase difference along the span, the fluctuating lift force acting on the cylinder $F_{L}$ ' is proportional to $C_{L}$ ' and is expressed as

$$
F_{L}^{\prime}=0.5 \rho u_{0}^{2} A C_{L}^{\prime},
$$

If there is a phase difference, the fluctuating lift reduces to

$$
F_{L}^{\prime}=\gamma\left(0.5 \rho u_{0}^{2} A\right) C_{L}^{\prime} \quad(0 \leq \gamma \leq 1)
$$

where $\gamma$ is the attenuation ratio due to the phase difference. Assuming a sufficiently long cylinder, $\gamma$ is expressed ${ }^{(15)}$ as

$$
\begin{aligned}
& \gamma=\frac{\sqrt{2 \Lambda(L-\sigma)}}{L}, \\
& \Lambda=\int_{0}^{\infty} R_{L L}(z) d z, \\
& \sigma=\frac{1}{\Lambda} \int_{0}^{\infty} z R_{L L}(z) d z,
\end{aligned}
$$

where $\Lambda$ is the spanwise correlation length, $\sigma$ is the centroid of the spanwise correlation, and $R_{L L}(z)$ is the cross-correlation coefficient between local fluctuating lift forces separated

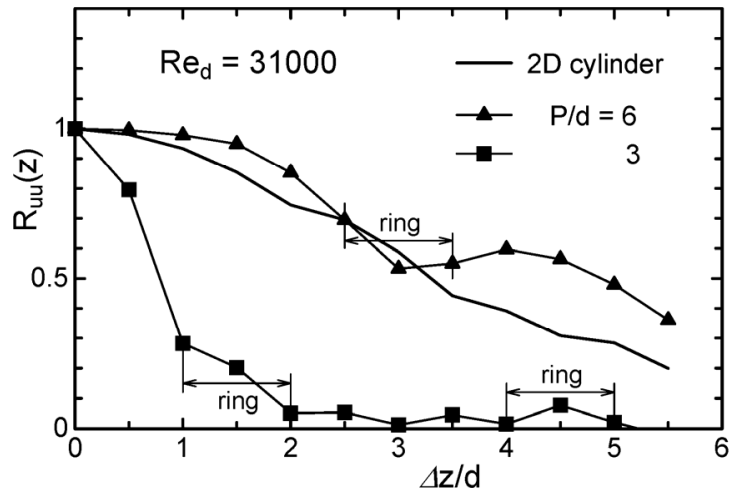

Fig. 8 Cross-correlation coefficient of the velocity fluctuation against spanwise separation

$$
\left(x=0, y / d=0.8, R_{d}=31,000\right)
$$


by a spanwise distance $z$. The value of $R_{L L}(z)$ can be approximated by the cross-correlation coefficient between local fluctuating velocities, $R_{u u}(z)$, which is calculated as

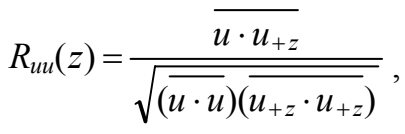

Figure 8 shows $R_{u u}(z)$ at $R e_{d}=31,000$ as a function of spanwise separation, $\Delta z$, which is the distance from the center of the base cylinder $(z=P / 2)$. The velocity fluctuation was measured using two I-type hot wires at the side of the cylinder $(x=0, y / d=0.8)$. The velocity fluctuation signal was band-passed within $f_{s} \pm 10 \%$ to estimate the value related to the suppression of periodic shedding.

For $P / d=6$, the spanwise correlation is similar to that of the $2 \mathrm{D}$ cylinder; the coefficient, however, increases slightly beyond the ring $(\Delta z / d \approx 4)$ since the periodic shedding phase tends to synchronize; the reason for this is not presently clear. In contrast, the spanwise correlation for $P / d=3$ decreases suddenly with $\Delta z / d$ since the periodicity disappears; the coefficient is almost zero beyond the ring $(\Delta z / d \geq 2)$.

Table 1 summarizes the estimated fluctuating lift force, $F_{L}{ }^{\prime}\left(f_{s}\right)$. For $P / d=3$, the value of $F_{L}{ }^{\prime}\left(f_{s}\right)$ is about $1 / 30$ that of the $2 \mathrm{D}$ cylinder due to the reduction of both the local fluctuating lift coefficient, $C_{L}$ ' $\left(f_{s}\right)$, and the spanwise correlation (reduction of $\gamma$ ).

Table 1 Estimation of fluctuating lift force $\left(D / d=1.3, W / d=1.0, L / d=18, \operatorname{Re}_{d}=31,000\right)$.

\begin{tabular}{ccccc}
\hline \hline & $C_{L}{ }^{\prime}\left(f_{s}\right)$ & $\gamma$ & $A / A_{2 \mathrm{D}}$ & $F_{L}{ }^{\prime}\left(f_{s}\right) / F_{L}{ }^{\prime}{ }_{2 \mathrm{D}}\left(f_{s}\right)$ \\
\hline 2D & 0.443 & 0.589 & 1.00 & 1.000 \\
$P / d=6$ & 0.141 & 0.636 & 1.05 & 0.362 \\
$P / d=3$ & 0.023 & 0.327 & 1.10 & 0.032 \\
\hline \hline
\end{tabular}

\section{A Consideration of the Mechanism}

\subsection{The change in flow around the ring}

The drag coefficient of the ringed cylinder decreases sharply above a Reynolds number of about 10,000, as shown in Fig. 2. This is caused by a change in flow around the ring, which leads to a narrowing of the wake behind the ring ${ }^{(13)}$. To explore the mechanism behind this, the flow field around the ringed cylinder was visualized.

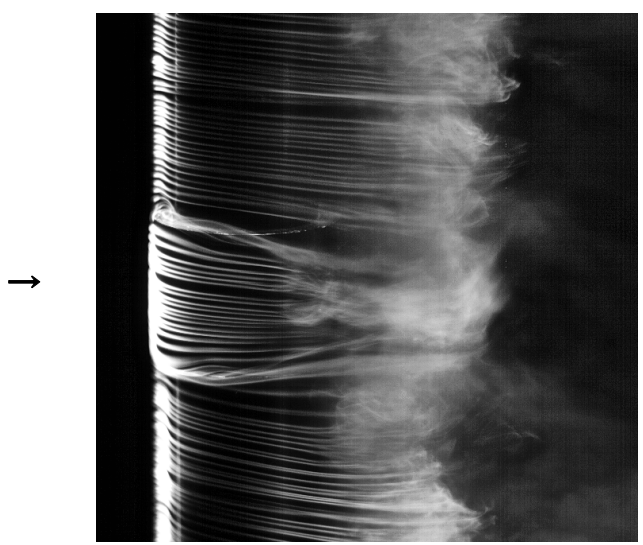

(a) $R e_{d}=10,000$

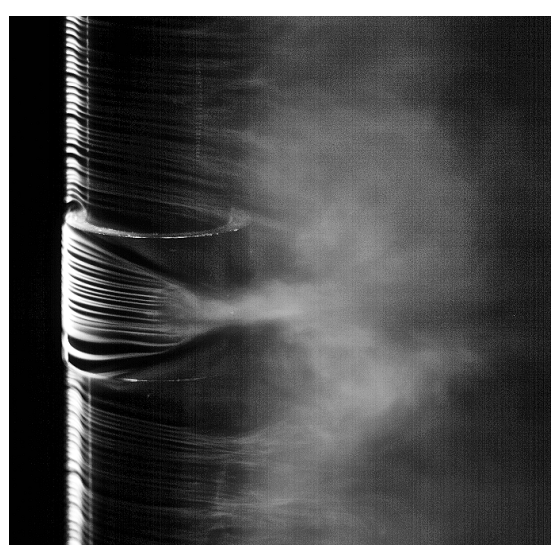

(b) $R e_{d}=20,000$

Fig. 9 Smoke visualization around the ringed cylinder for $P / d=3$ $(D / d=1.3, W / d=1)$; wire parallel to the $z$ axis at $x / d=-0.7, y / d=-0.05$ 
Figure 9 shows the flow around the ringed cylinder $(D / d=1.3, W / d=1, P / d=3)$ visualized by the smoke-wire method. A tightened $0.1 \mathrm{~mm}$ Nichrome wire was placed just upstream of the cylinder $(x / d=-0.7, y / d=-0.05)$ in the spanwise direction. As shown in Fig. 9, the flow on the side of the ring shrinks in the spanwise direction; this is because the velocity just outside of the boundary layer on the side of the ring is generally higher than that on the side of the base cylinder at the same radius. This causes a spanwise pressure differential and results in flow from the base cylinder (near the ring edge) to the side of the ring. This flow even appears at a lower Reynolds number of $R e_{d}=10,000$ (Fig. 9[a]) but becomes prominent at $R e_{d}=20,000$ (Fig. 9[b]).

Figure 10 shows the power spectrum of the velocity fluctuation at the side of the ring $(y / d=0.8)$. At $R e_{d}=10,000$ (Fig. 10[a]), the turbulence level around the ring is similar level to that for the $2 \mathrm{D}$ cylinder. In contrast, at $R e_{d}=31,000$ (Fig. $10[\mathrm{~b}]$ ), the turbulence level markedly increases at the ring edge $(z / d=0.5)$ for the higher frequency components. (The turbulence level already increases at $R e_{d}=20,000$ according to Ref.[13]). This indicates that laminar-turbulent transition initiates at the ring edge in the range of $10,000<R e_{d}<20,000$.

For a 2D circular cylinder, it is well known that the shear layer undergoes laminar-turbulent transition in the critical regime $\left(3 \times 10^{5}<R e_{d}<1.5 \times 10^{6}\right)$ and the flow reattaches just behind the separation line, forming a separation bubble between the separation and reattachment points. Similarly, for the ringed cylinder, it is reasonable to suppose that the transition at the ring edge at $R e_{d}>10000$ triggers the flow reattachment at the rear of the ring to form a separation bubble on the side of the ring, despite the Reynolds number being more than an order of magnitude lower.

The mechanism behind this can be considered as follows: At the ring edge, a corner

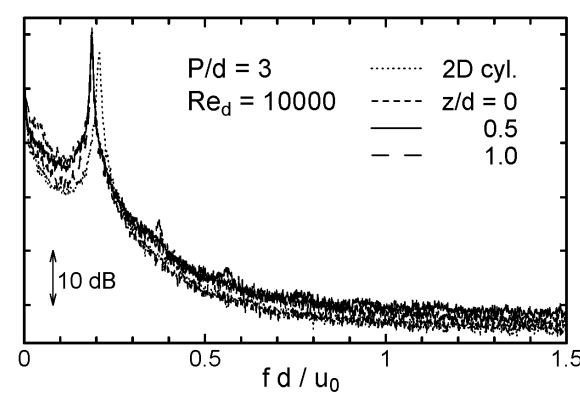

(a) $R e_{d}=10,000$

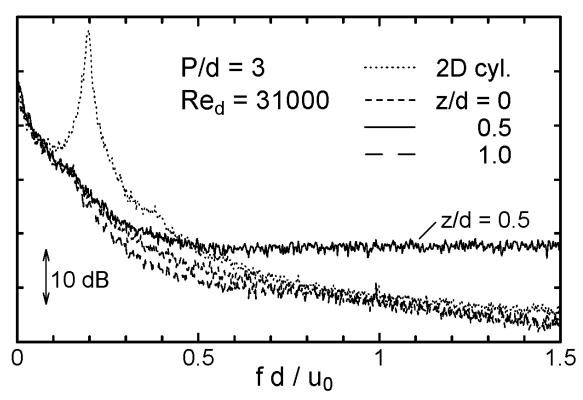

(b) $R e_{d}=31,000$

Fig. 10 Power spectrum of the velocity fluctuation at the side of the cylinder measured by an I-type hot wire at $x=0, y / d=0.8(P / d=6, D / d=1.3, W / d=1)$

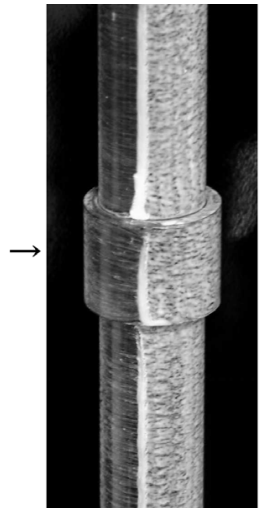

(a) $R e_{d}=10,000$

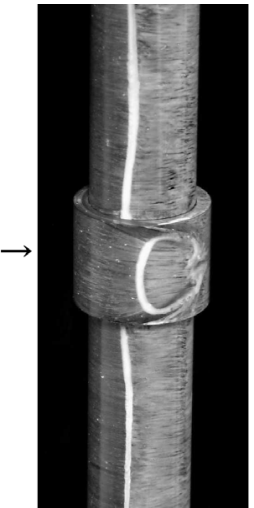

(b) $R e_{d}=20,000$

Fig. 11 Surface oil-film pattern around the ring

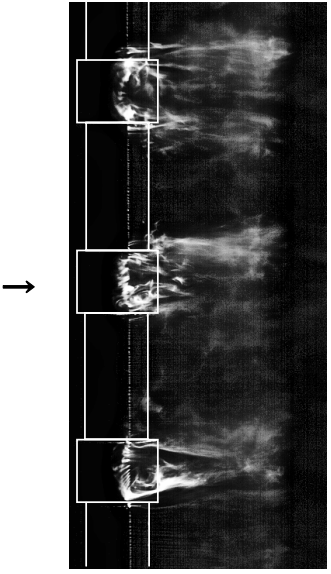

(a) $R e_{d}=10,000$

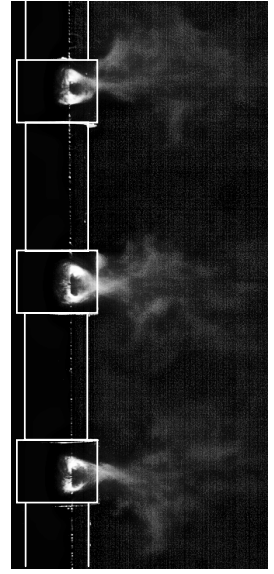

(b) $R e_{d}=20,000$

Fig. 12 Smoke visualization around the ring 
vortex is generated from the front to the side of the cylinder, as clearly visualized in Fig. 9(a), and is entrained in the flow on the side of the ring due to the pressure differential. This causes a higher turbulence level in the separated shear layer around the ring edge, which promotes turbulent transition at lower Reynolds numbers. Once the separation bubble is formed, the surface pressure on the side of the ring decreases markedly, as shown in Fig. 6(a). This intensifies the flow narrowing in the spanwise direction at the side of the ring, as shown in Fig. 9(b), which again induces turbulent transition. This synergistic effect maintains the existence of the separation bubble.

The separation bubble is depicted in Figs. 11 and 12. Figure 11 shows the surface oil-film patterns on the side of the ring at the same condition as in Fig. 9. At $R e_{d}=10,000$ (Fig. 11[a]), the flow separation line on the side of the ring is almost two-dimensional; however, at $R e_{d}=20,000$ (Fig. 11[b]), the separation line is formed into a C-shape by the flow shrinkage in the spanwise direction. The flow reattachment point is also visible near the mid-span of the ring at $\phi \approx 140^{\circ}$.

Figure 12 shows the flow visualization using the smoke-wire method on the side of the ring. The wire was placed $1 \mathrm{~mm}$ from the ring surface at $\phi=125^{\circ}$. For both Reynolds numbers $\left(R e_{d}=10,000\right.$ and 20,000), a reverse flow forms on the ring from the wire to just behind the flow separation line, and then the flow spreads to both ring edges along the separation line. At $R e_{d}=10,000$ (Fig. 12[a]), the spread reverse flow separates and entrains in the wake of the cylinder, forming a quasi-two-dimensional spanwise vortex behind the cylinder. On the other hand, at $R e_{d}=20,000$ (Fig. 12[b]), the spread reverse flow merges near the mid-span of the ring at $\phi \approx 140^{\circ}$ and then separates further downstream. The C-shaped separation bubble is clearly visible on the side of the ring, corresponding to the oil-film pattern shown in Fig. 11(b). In this case, the quasi-two-dimensional spanwise vortex is not observed in the wake.
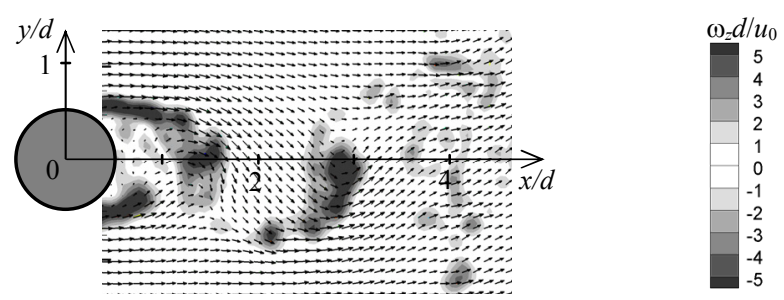

(a) 2D cylinder
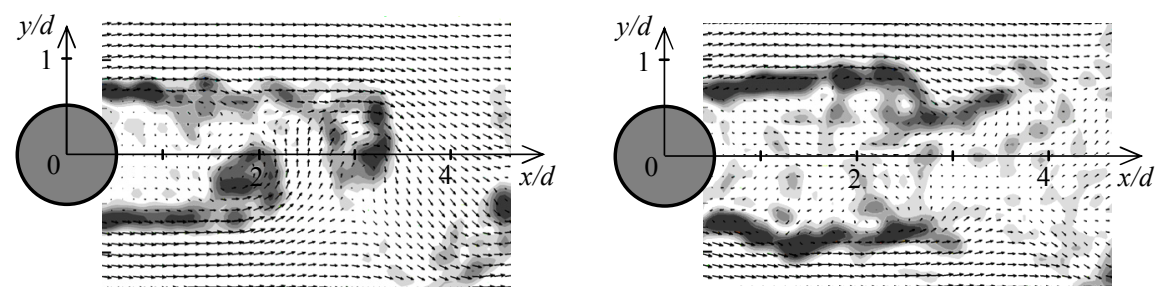

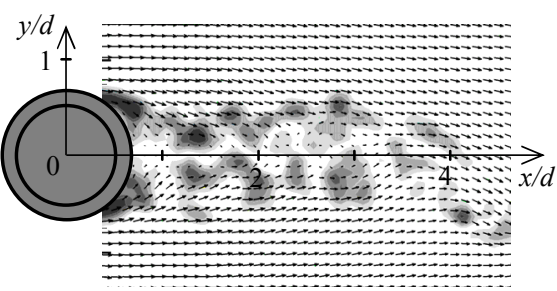

(b) $P / d=6$

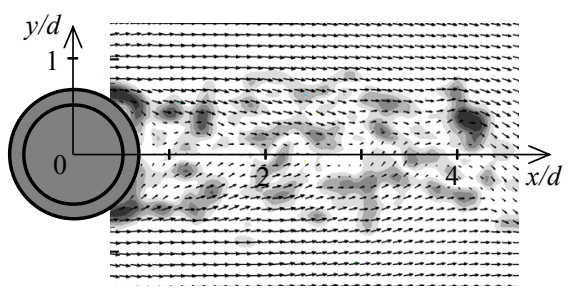

(c) $P / d=3$

Fig. 13 Instantaneous velocity field and vorticity contour behind the cylinder in the $x-y$ plane at $R e_{d}=$ $30,000(D / d=1.3, W / d=1)$; top - behind the base cylinder at $z=P / 2$, bottom - behind the ring at $z=0$ 


\subsection{Velocity field after wake transition}

Next, the flow field behind the ringed cylinder was measured using PIV. Figure 13 depicts an instantaneous velocity field and vorticity contour behind the cylinder in the $x-y$ plane at $R e_{d}=30,000$ (after transition). For the 2D cylinder (Fig. 13[a]), alternating vortex shedding generates a mass of vorticity across the wake center $(y=0)$ just behind the cylinder. This induces periodic transverse flow near the cylinder, causing a significant amount of fluctuating lift. In contrast, for the ringed cylinder, the wake behind the ring (Fig. $13[\mathrm{~b}]$ and [c], bottom) shrinks due to the formation of separation bubbles on both sides of the ring. The small-scale vortices are shed behind the ring, but they barely cross the wake center. For $P / d=6$ (Fig. 13[b], top), the vortex formation region moves downstream on the base cylinder, causing periodic transverse motion near the cylinder to weaken, which then leads to a reduction in fluctuating lift. The smaller pitch configuration further weakens the periodic motion, and finally, periodic shedding ceases at $P / d \approx 3$ (Fig. 13 [c], top).

The three-dimensionality of the wake is depicted in Fig. 14, which shows the mean velocity field behind the cylinder at the wake center $(x-z$ plane, $y=0)$. For the 2D cylinder (Fig. 14[a]), the mean velocity field is uniform along the span, although there is a three-dimensionality to the instantaneous field, and a reverse flow forms just behind the cylinder $(x / d<1.3)$. In contrast, the addition of the ring produces a pair of transverse vortices $\left( \pm \omega_{y}\right)$ just behind the ring edge, as indicated by the arrows in Fig. 14(b) and (c); this is because the flow behind the ring has no reverse flow in the mean field, while the flow behind the base cylinder has a large reverse flow region for $x / d<3$. This distorts the wake around the ring into a highly three-dimensional feature. For the larger ring pitch of $P / d=6$ (Fig. 14[b]), there remains a two-dimensional flow field behind the base cylinder. This maintains periodicity in the vortex shedding at the middle of the base cylinder (Fig. 4[b], left). With decreasing ring pitch, the two-dimensional flow field behind the base cylinder shortens until it disappears at $P / d \approx 3$, as shown in Fig. 14(c). This suppresses the periodicity of the vortex shedding.

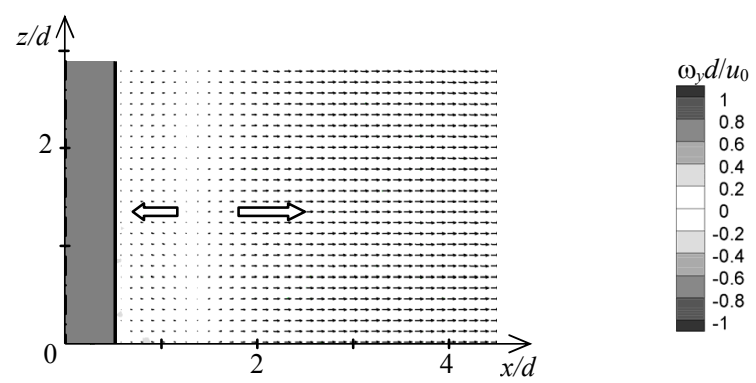

(a) $2 \mathrm{D}$ cylinder

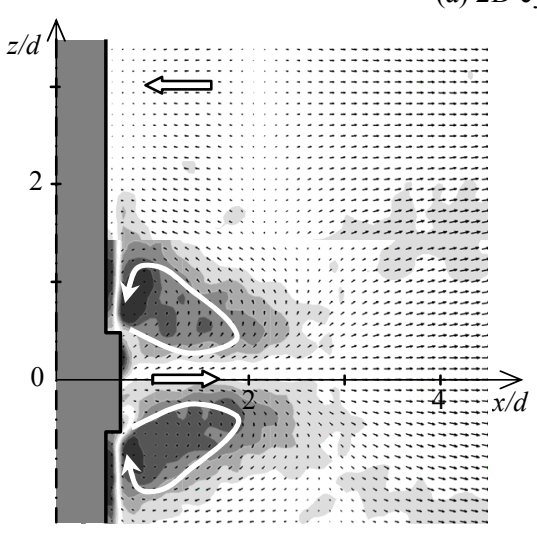

(b) $P / d=6$

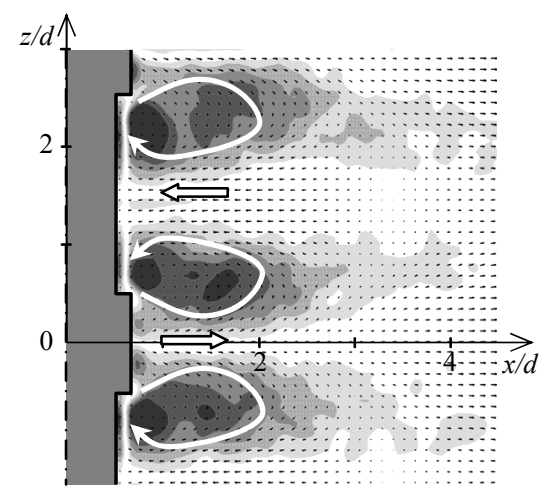

(c) $P / d=3$

Fig. 14 Mean velocity field and vorticity contour behind the cylinder at the wake center $(x-z$ plane, $y=0)$ at $R e_{d}=30,000(D / d=1.3, W / d=1)$ 


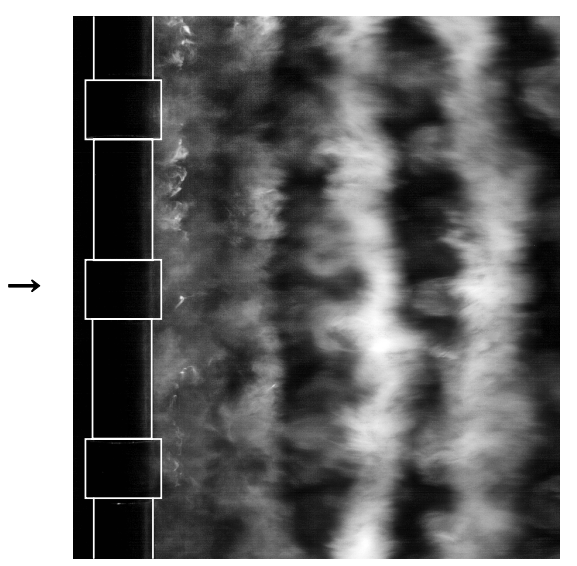

(a) $R e_{d}=10,000$

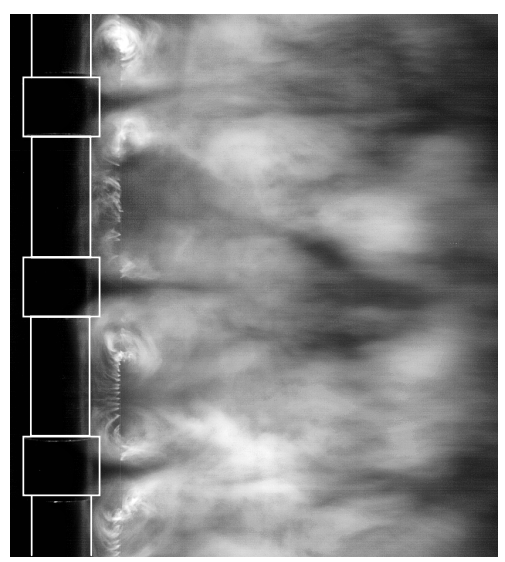

(b) $R e_{d}=20,000$

Fig. 15 Smoke visualization behind the ringed cylinder for $P / d=3(D / d=1.3, W / d=1)$; wire parallel to the $z$ axis at $x / d=1, y / d=0$

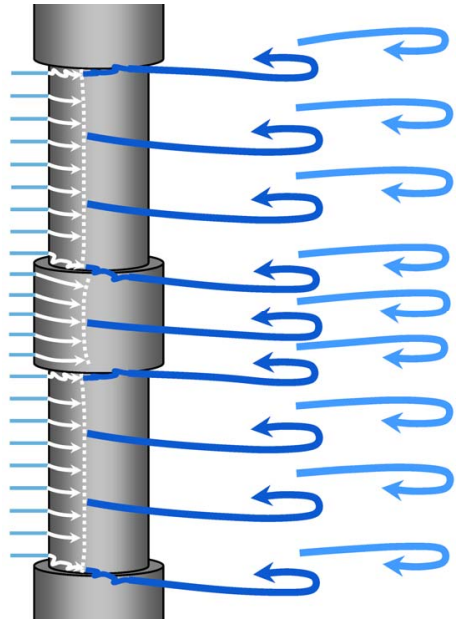

(a) $R e_{d}=10,000$

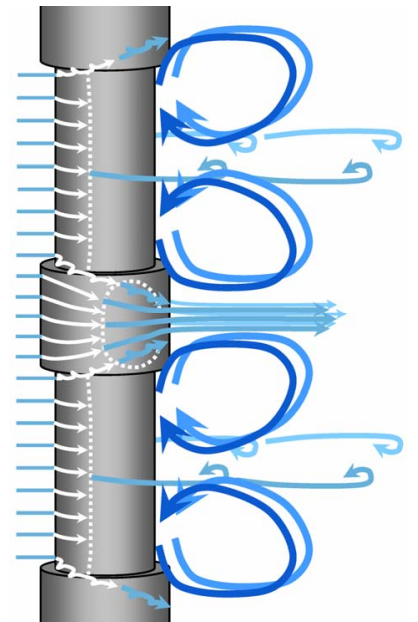

(b) $R e_{d}=20,000$

Fig. 16 Conceptual flow structure around the ringed cylinder for $P / d \approx 3$

\subsection{Change in the vortical structure of the wake}

Figure 15 shows a smoke visualization of the wake center $(y=0)$ at the same conditions as in Fig. $9(D / d=1.3, W / d=1, P / d=3)$. For $R e_{d}=10,000$, the rings cause local three-dimensional flow (Fig. 9[a]). However, the spanwise vortices generated in the separated shear layer tend to connect in the near wake, as can be observed in Fig. 12(a). Finally, a quasi-two-dimensional spanwise vortex tube is formed as shown in Fig. 15(a), resulting in periodic vortex shedding similar to that in the $2 \mathrm{D}$ cylinder.

In contrast, for $R e_{d}=20,000$ (Fig. 15[b]), a pair of transverse vortices just behind the ring edges are clearly visible, which correspond to $\pm \omega_{y}$ measured using PIV (Fig. 14[c]). The movie of Fig. 15(b) shows that the transverse vortices rotate continuously, the position of which is almost fixed. This flow obstructs the formation of two-dimensional spanwise vortices.

Finally, the flow structures around the ringed cylinder before and after the transition are conceptually illustrated in Fig. 16. For $R e_{d} \leq 10,000$ (Fig. 16[a]), the spanwise flow shrinks on the side of the ring due to the pressure differential, which entrains the corner vortices at the ring edge to the side of the ring. However, the turbulence level of the shear layer separated from the ring is not high enough to cause turbulent transition, and thus quasi-two-dimensional spanwise vortices are formed in the wake, leading to periodic 
shedding. In contrast, for $R e_{d} \geq 20,000$ (Fig. 16[b]), the turbulent transition occurs in the shear layer separated from the ring, forming separation bubbles on both sides of the ring. This shrinks the wake behind the ring, which then forms a pair of transverse vortices behind the ring edges. This flow alternation obstructs the formation of spanwise vortices, thus suppressing periodic shedding.

\section{Conclusions}

To investigate vortex shedding suppression by the addition of cylindrical rings to a circular cylinder, experiments were performed at Reynolds numbers ranging between $R e_{d}=$ 10,000 and 40,000. It was found that fluctuating lift almost disappeared at $R e_{d} \geq 20,000$ for the ring configuration of $D / d=1.3, W / d=1$, and $P / d \approx 3$. This was caused by the reduction in both the local fluctuating lift coefficient and the spanwise correlation.

A scenario for vortex shedding suppression was considered as follows: A spanwise pressure gradient originating from a stepwise change in diameter induces a spanwise flow, which brings the corner vortex at the ring edge to the side of the ring. This promotes turbulent transition in the shear layer separated from the side of the ring at $R e_{d} \geq 20,000$. As a result, the separated flow reattaches at the rear of the ring to form a separation bubble, which shrinks the wake behind the ring; this induces a pair of large transverse vortices just behind the ring edges. Consequently, this flow alternation obstructs the formation of two-dimensional spanwise vortices and suppresses periodic vortex shedding.

\section{Acknowledgements}

The author wishes to acknowledge Ms. Mariko Masuda, Mr. Taichi Okada, Mr. Naoki Shiibara, Mr. Wataru Kaneko, Mr. Kai Nakajima, and Mr. Yoichiro Adachi, former students at the National Defense Academy of Japan, for their assistance in the experiments and data analysis.

\section{References}

(1) Zdravkovich, M., Review and Classification of Various Aerodynamic and Hydrodynamic Means for Suppressing Vortex Shedding, J. Wind Engineering and Industrial Aerodynamics, Vol.7 (1981), pp. 145-189.

(2) Fage, A., and Warsap, J.H., The effects of Turbulence and Surface Roughness on Drag of a Circular Cylinder, Br. Aerodyn. Res. Counc., Rep. Memo. (1929) 1283.

(3) James D.F., and Truong, Q.S., Wind Load on Cylinder with Spanwise Protrusion, Proc. Am. Soc. Civ. Eng., J. Eng. Mech. Div., 98 (1972), pp.1573-1589.

(4) Bearman, P. W. and Owen, J. C., Reduction of bluff-body drag and suppression of vortex shedding by the introduction of wavy separation lines, J. Fluids and Structures Vol.2, (1998), pp.123-130.

(5) Owen, J. C., Bearman, P. W. and Szewczyk, A. A., Passive control of VIV with drag reduction, J. Fluids and Structures Vol.15 (2001), pp.597-605.

(6) Scruton, C., and Walshe, D.E.J., A Means for Avoiding Wind-Excited Oscillations of Structures with Circular or Nearly Circular Cross Section, Natl, Phys. Lab. (U.K.), Aero Rep. (1957), 335.

(7) Nakagawa, K., Fujino, T., Arita, Y. and Shima, K, An Experimental Study of Aerodynamic Devices for Reducing Wind-Induced Oscillatory Tendencies of Stacks, Proc. Symp. Wind Effects on Buildings and Structures, Teddington (1963), pp. 774-795.

(8) Ahmed, A. and Bays-Muchmore, B., Transverse Flow Over a Wavy Cylinder, Phys. Fluids A Vol.4 No.9 (1992), pp.1959-1967. 
(9) Ahmed, A., Khan, M.J. and Bays-Muchmore, B., Experimental Investigation of a Three-Dimensional Bluff-Body Wake, AIAA Journal, Vol.31 No.3 (1993), pp.559-563.

(10) Lam, K., Wang, F. H., Li, J. Y. and So, R. M. C., Experimental Investigation of the Mean and Fluctuating Forces of Wavy (Varicose) Cylinders in a Cross-Flow, J. Fluids and Structures Vol.19 (2004), pp.321-334.

(11) Zhang, W. and Lee, S. J., PIV Measurements of the Near-Wake behind a Sinusoidal Cylinder, Experiments in Fluids Vol.38 (2005), pp.824-832.

(12) Nakamura, H and Igarashi, T., Omni-Directional Drag Reduction of a Circular Cylinder by Attaching Rings, Proc. 5th Int. Colloquium on Bluff Body Aerodynamics \& Applications, Ottawa (2004), pp.163-166.

(13) Nakamura, H. and Igarashi, T., Reductions in Drag and Fluctuating Forces for a Circular Cylinder by Attaching Cylindrical Rings, J. Fluid Science and Technology Vol.2 No.1 (2007), pp.12-22.

(14) Lim, H. C. and Lee, S. J., Flow Control of a Circular Cylinder with O-Rings, Fluid Dynamic Research Vol.35 (2004), pp.107-122.

(15) Norberg, C., Fluctuating Lift on a Circular Cylinder: Review and New Measurements, J. Fluids and Structures Vol.17 (2003), pp.57-96.

(16) Kiya, M., Suzuki, Y. Arie, M. and Hagino, M., A Contribution to the Free-Stream Turbulence Effect on the Flow Past a Circular Cylinder, J. Fluid Mech. Vol.115 (1982), pp.151-164. 